\title{
Guideline for comparing functional enrichment of biological network modular structures
}

\author{
Guillermo de Anda-Jáuregui (D)
}

Correspondence: gdeanda@inmegen.edu.mx Computational Genomics, Instituto Nacional de Medicina Genómica, Periférico Sur 4809, Ciudad de México, México

\begin{abstract}
The use of networks to analyze biological data, such as large gene or protein expression datasets, is on the rise. Often, there is an interest of identifying modules (or communities) of biological molecules that may be associated to known functions. This functional modularity analyses usually revolve around a workflow that combines 1) a method for network reconstruction from biological data, 2) a community or clustering algorithm on a network, and 3) an enrichment analysis to associate modules to known biological categories. With this, it is possible to identify sets of functions associated to modules in networks of distinct biological conditions, allowing for the comparison of such different phenotypes.

Currently there is no set of recommendations for such analyses, which can lead to problems in assessing these results for a given biological context. Furthermore, without properly identifying the methodological scopes and limitations at each stage for a given functional modularity analysis, it is not immediately possible to compare the biological implications of analyses in different phenotypes.

In this work, critical points in a functional modularity analysis for biological networks are identified, and methods are proposed for assessing the topological and biological results of functional modularity analyses in biological networks, and to calculate topological and functional similarity between comparable phenotypes. These methods are demonstrated on biological networks artificially constructed from known biological pathways.
\end{abstract}

Keywords: Communities, Modularity, Biological networks, Functional enrichment

\section{Background}

The emergence of high-throughput technologies for the study of biological systems has lead to the use of several data-centric strategies for their study. Among these, techniques derived from network science are increasingly being adopted (Ma'ayan 2011; Dobrescu and Purcarea 2009; Barabási and Oltvai 2004). A well described property of biological systems is their modular structure (Lorenz et al. 2011), with different biological functions being controlled by different sets of molecular interactions (Ames et al. 2013).

As biological network analysis becomes an increasingly important tool in the study of biological systems, the detection of modular structures in this networks, and module enrichment (the association of these modules or communities to known biological functions) becomes more commonplace (Alcalá-Corona et al. 2017; Langfelder and Horvath

(C) The Author(s). 2019 Open Access This article is distributed under the terms of the Creative Commons Attribution 4.0 International License (http://creativecommons.org/licenses/by/4.0/), which permits unrestricted use, distribution, and reproduction in any medium, provided you give appropriate credit to the original author(s) and the source, provide a link to the Creative Commons license, and indicate if changes were made. 
2008; Liu et al. 2017; Adamcsek et al. 2006). A PubMed query for networks, clusters, modules, and enrichment returns over 900 hits, with 222 from the year 2018 alone (see Additional file 1: Figure S1 for an illustration). As this trend continues, it will be important for biological and biomedical researchers to identify critical points in the workflows used for such analyses.

\section{Critical points in a typical modular analysis of a biological network}

There are many different methodological strategies available for the construction of networks from biological data, for the identification of biological communities or modules in these networks, and for the association of known biological functions to these modules of biomolecules). In general, these analysis pipelines will consist of three steps:

1. A methodology for network construction: these include the integration of known biomolecular interactions from available literature (Hur et al. 2009) or public databases such as The Kyoto Encyclopedia of Genes and Genomes (KEGG) (Kanehisa and Goto 2000) or the Search Tool for the Retrieval of Interacting Genes database (STRING-db) (Szklarczyk et al. 2017), or probabilistic methods of gene or protein co-expression inference (Langfelder and Horvath 2008; Margolin et al. 2006; Madhamshettiwar et al. 2012).

2. An algorithm for the identification of communities or modules in the network: there is an abundant body of published algorithms for the detection of communities in large complex networks based on different approaches (Fortunato 2010).

3. An enrichment analysis to associate the modules to known biological processes or functions: There is a variety of methods using different approaches

(García-Campos et al. 2015) that may be used to associate, the sets of biomolecules that form each module to sets of biomolecules that are known to be involved in biological functions, such as those described in databases like the aforementioned KEGG or the Gene Ontology (Consortium 2015).

With many different combinations of methodologies available for these module enrichment studies, it becomes difficult to assess whether the results obtained through one pipeline are comparable to results obtained from another. In this work, we will find parameters that allow for the quantitative comparison of modular structures of biological networks from a topological and functional perspective.

\section{Data}

In thiswork, we use four different biological networks, with nodes representing genes. These networks are constructed by merging different pathways obtained from the Kyoto Encyclopedia of Genes and Genomes (KEGG) (Kanehisa and Goto 2000) into undirected, unweighted graphs. A visualization of this networks is found in Fig. 1.

We will describe different measures to compare these networks and their modular structures from a topological and functional perspective. For this, we will identify each network as follows:

1. The first network is the Main network. It is constructed by merging 5 pathways (as mentioned in "Methods"). This will be the network against which other networks will be compared. 


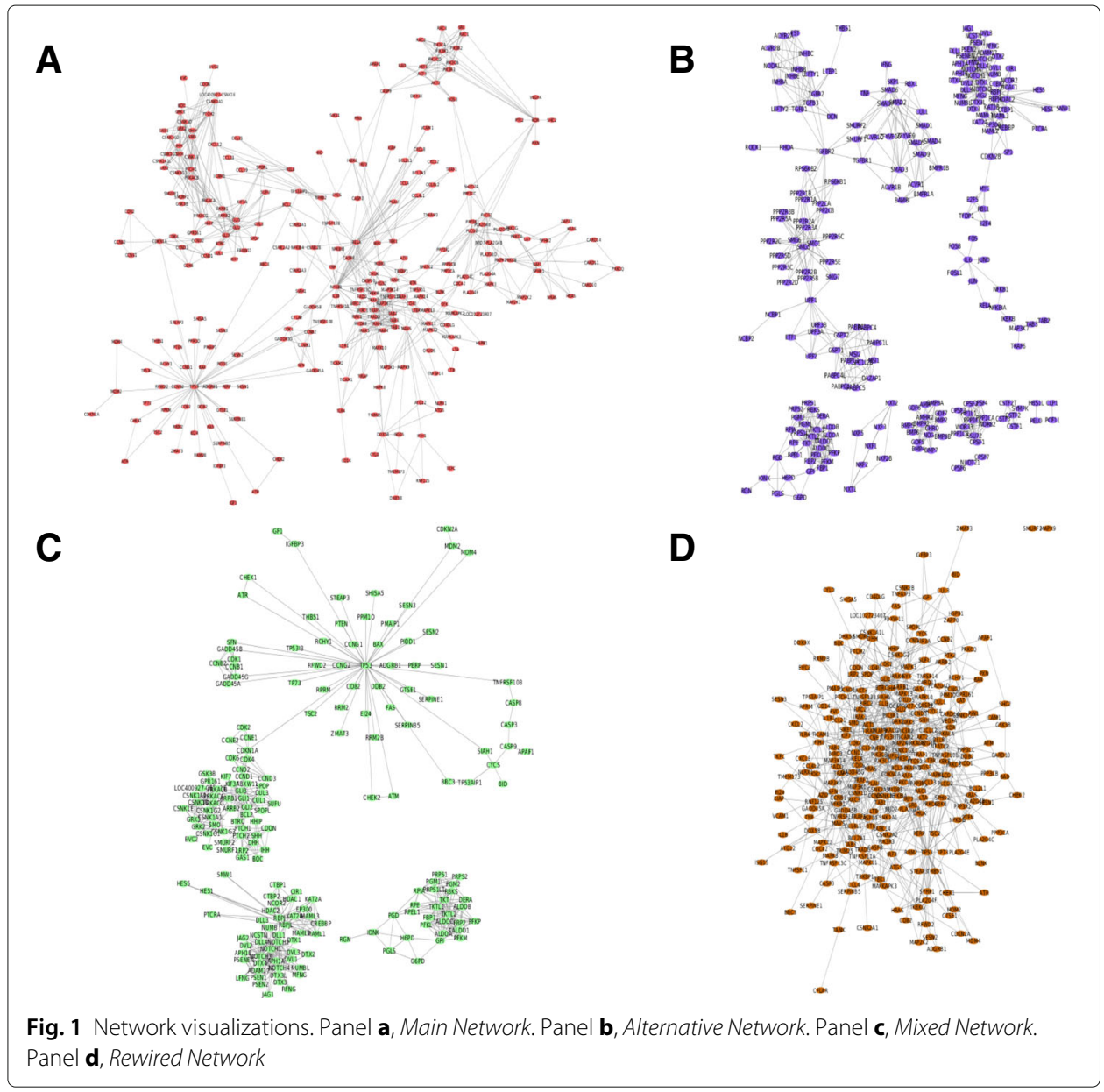

2. The second network is the Alternative network. It was constructed from pathways with almost no overlap to the ones used to build the Main network.

3. The third network is the Mixed network, constructed from two pathways from Main and two pathways from Alternative.

4. The last network is a Rewired network derived from the Main network.

\section{Modular structure comparison pipeline}

Consider a pipeline for the comparison of modular structures in biological networks, containing the aforementioned critical points. In Fig. 2, a graphical representation of this proposed pipeline is shown, indicating the quantitative parameters that may be used for the comparison of networks at each level. In what follows, we will explore each of these comparison levels, using a set of artificial networks generated from known biological pathways, which attempt to represent likely structures of networks generated from biological data.

\section{Network topological comparison}

The first critical step is to assess the comparability of biological networks. To do so we must describe the topological features of these networks. Since a common use-case for 


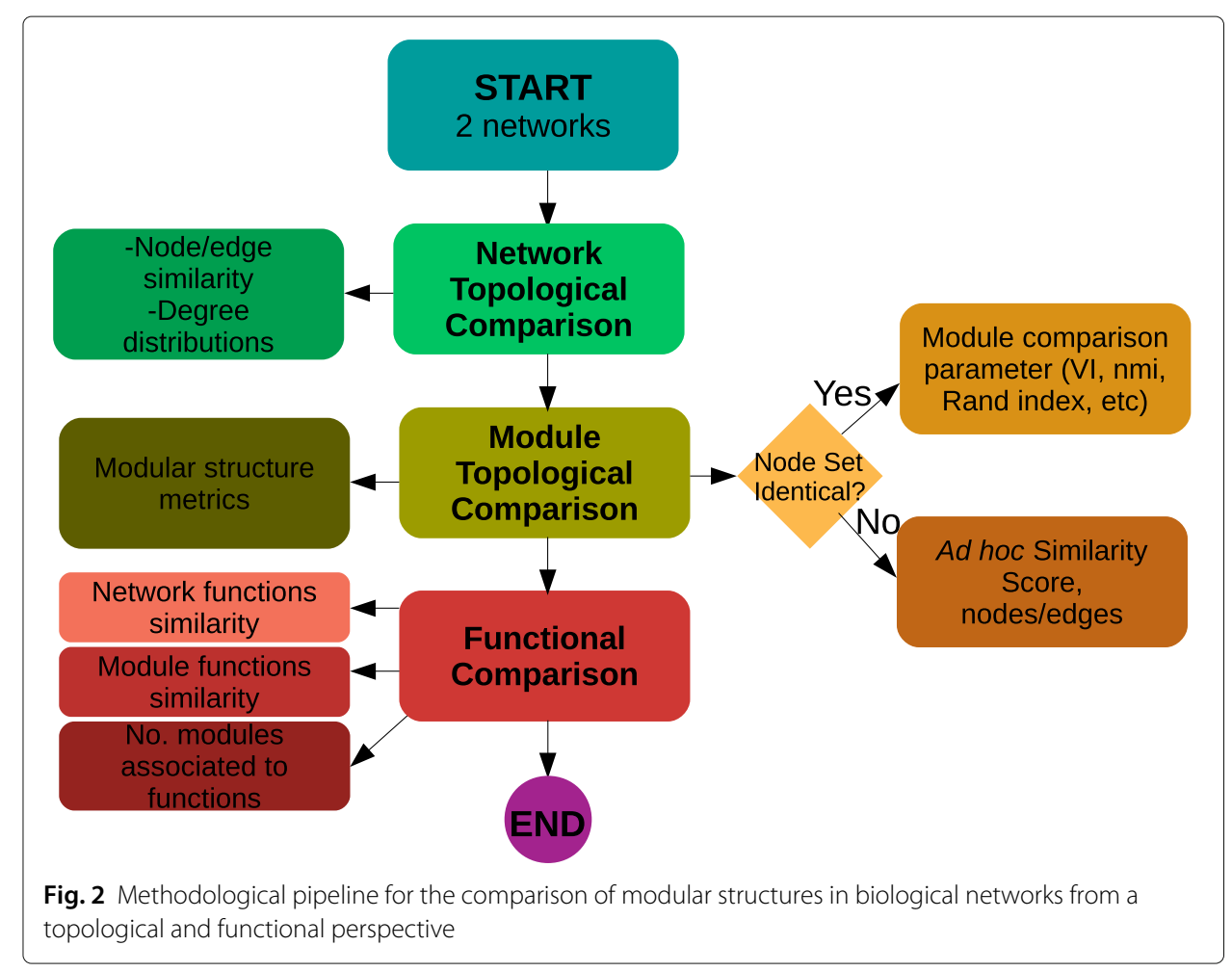

network analyses in the biological sciences is data exploration, these descriptors are readily available. Importantly, these features will be directly related to the way the networks are originated: whether they are curated from literature, inferred using a probabilistic approach from high throughput data, reconstructed through experimental methods, etc. The decision on whether networks are comparable based on their structural patterns can only be assessed based on the biological question of interest. For instance:

- Two sets of genes associated to two different biological conditions are used as the bases for network construction; these gene sets may have different sizes, and therefore the number of nodes will be different.

- A method for the inference of whole-genome co-expression network is used for two different biological conditions; the set of associated co-expression relationships may differ between the two conditions.

A researcher shall consider, based on their originating data and the biological question at hand, at what level the networks are comparable. Furthermore, the results and biological insights derived from these networks shall be explicitly described in this context.

By construction, we generated two networks (Alternative and Mixed networks) that are different from our Main Network in terms of number of nodes and edges. Meanwhile, we generated a Rewired network that has the same number of both nodes and edges. Some basic descriptors of these networks are found in Table 1. Furthermore, none of the networks have the same degreedistribution, as illustrated in Fig. 3.

For the purposes of functional comparability of nodes, perhaps the most immediate parameter to consider is the similarity in number of nodes, as this will be a decision point 
Table 1 Basic Network Descriptors

\begin{tabular}{lllll}
\hline & Main & Alternative & Mixed & Rewired \\
\hline No.Nodes & 276 & 228 & 189 & 276 \\
No.Edges & 692 & 723 & 596 & 692 \\
No.Components & 1 & 11 & 3 & 3 \\
\hline
\end{tabular}

in further comparison. Differences in the number of edges are however, quite important in the context of module analysis, as these modules are defined not only by nodes but by the connections between them. This consideration could be extended to the influence of a difference in component number, as a network with more than one component will, by definition, have at least one pair of nodes without a linking path.

The biological network structures associated to different phenotypes will hopefully reflect the differences between the underlying biological phenomena. The modular structure will inherit from these network structures. This shall be kept in mind while discussing the results of further analyses.

\section{Node set identity comparison}

It is important to assess not only the topological similarity of networks, but whether the biological elements that are represented in the networks are comparable. In order to do this, a similarity measure is used to compare the identity (ie, labels) of nodes in different graphs. For instance, we could use the Jaccard index $(J)$, as seen in Table 2. Jaccard index and other measures used throughout the manuscript are defined in the "Methods" section.

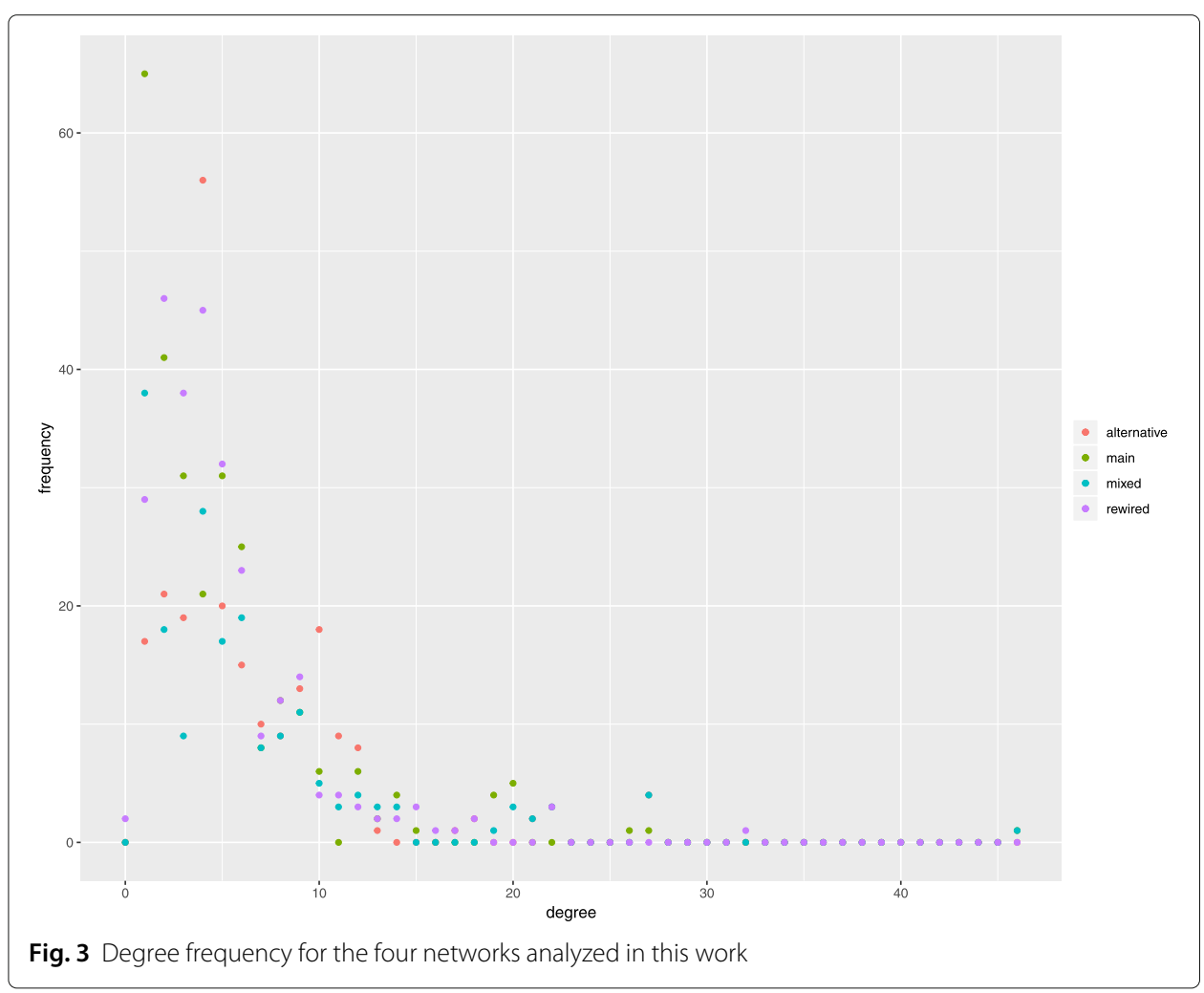


Table 2 Node similarity, compared to the Main network

\begin{tabular}{ll}
\hline & \\
\hline Main & Jaccard Index, nodes \\
Alternative & 1.00 \\
Mixed & 0.03 \\
Rewired & 0.32 \\
\hline
\end{tabular}

The rewired network has exactly the same set of nodes representing genes, as it was generated by reconnecting the Main network, whereas the others are dissimilar to the original one. It can be noticed that the alternative network is not completely dissimilar to the original one (as one may naively guess), since there is a small overlap in the genes composing the pathways used for the construction of the Main and alternative networks (13 genes in total). Edge similarity can also be evaluated, as seen in Table 3.

Again, a similarity threshold for nodes and edges must be defined in terms of the biological question that is being studied. For instance, a biological question in itself could be if networks reconstructed from two different conditions using the same methodology is composed of the same biological molecules. In other cases, it is necessary for the networks to be composed by the same set of nodes. For the purposes of module comparison, whether the node set of each network to be compared is a critical point that defines which methods are available to compare the modular structures of two different networks.

\section{Comparison of modular structures}

Once the structural comparability of the networks has been assessed, it would be possible to analyze and compare the modular structures that are identified.

\section{Election of the module detection algorithm}

Selecting the module detection algorithm for a given analyses is an open problem (Jebabli et al. 2017). Ideally, the selected algorithm would be able to recover an organization of the constituent elements in the network that has its origin in a biological phenomenon. Assessing how suitable an algorithm is would then consist on evaluating how well it recovers such structures. Unfortunately, in the case of biological networks such ground truth is seldom available.

Comparing partitions generated by an algorithm to the ground truth organization is a problem that can be approached with different well described techniques. Several methods have been developed and implemented: these include Variation of Information (Meilă 2003), Normalized Mutual Information (Danon et al.), Adjusted Mutual Information (Vinh et al. 2009), split-join distance (van Dongen 2000), and (adjusted) Rand index (Rand 1971; Hubert and Arabie 1985). A description of these methods is beyond the scope of this manuscript (see Vinh et al. (2010) and Orman et al. (2012)), but are noted for such

Table 3 Edge similarity, compared to the Main network

\begin{tabular}{ll}
\hline & Jaccard Index, edges \\
\hline Main & 1.00 \\
Alternative & 0.00 \\
Mixed & 0.26 \\
Rewired & 0.37 \\
\hline
\end{tabular}


cases in which such ground truth was available. When such information is unavailable, it will be necessary to resort to heuristics, or to identify consensus between algorithms.

For this work, different algorithms were tried and the partitions generated compared to each other (see Additional file 2: Figure S2, in which different module detection algorithms are compared using Adjusted Mutual Information). For simplicity, throughout the manuscript the results of the Infomap algorithm will be used. The decision to use this algorithm was arbitrary, since the intention is to illustrate the critical points of the functional module analysis workflow.

\section{Evaluation and comparison of modular structures}

Having identified modular structures in each biological network, the question is how similar are these modular structures to each other. A first approach is to obtain and compare descriptors of these modular structures. The modularity value $Q$ (Newman and Girvan 2004; Clauset et al.) measures whether a proposed division of a network is adequate capturing communities, in the sense of recovering groups with a higher proportion of edges within the group than to other groups.

In the case of comparing different biological networks and their associated modular structures, by comparing the value of $Q$ for each network partition it is possible to assess if there is one network in which the module detection algorithm, applied to each network, identified a more modular structure. In Table 4, the results for the example networks are shown.

The measure of modularity $Q$ is readily available in network analysis packages. While widely used, there are limitations in the use of $Q$ as a sole measure of the modular structure of a network identified by a module detection algorithm: particularly, it should be noted that since this metric is used as an optimization criterion for certain module detection algorithms, it could bias comparisons (Jebabli et al. 2017). Further descriptors should be used in order to more adequately capture the differences in modular structures.

The node similarity of the networks is critical to decide how to compare modular structures. The methods available for networks with identical node sets will be different to those for networks composed of non-identical node sets.

\section{Comparison of modular structures in networks with identical node sets}

If the node sets of the networks to be compared are identical, then the problem of comparing their modular structures is not entirely unlike the previously described problem of comparing modular partitions on the same network derived from different algorithms: instead of comparing a partition of graph $G_{1}$ obtained by algorithm $A$ with the partition of $G_{1}$ obtained by algorithm $B$ (or perhaps to a ground truth partition), it would be a comparison of the partitions obtained using algorithm $A$ of graphs $G_{1}$ and $G_{2}$.

Table 4 Modularity of the Infomap Partition

\begin{tabular}{ll}
\hline & $\mathrm{Q}$ \\
\hline Main & 0.75 \\
Alternative & 0.85 \\
Mixed & 0.77 \\
Rewired & 0.48 \\
\hline
\end{tabular}


To do such comparison, the methods previously mentioned when discussing the election of module detection algorithms are available. For the purposes of this manuscript, two of the constructed networks were generated to satisfy the condition of containing the same set of nodes: the Main and Rewired networks. In Table 5, the use of the different comparison methods is illustrated, as applied to these networks.

By using these results it is possible to provide an overall descriptor of how different is the organization of elements in the network, derived from the differences in network structure, as identified by the module detection algorithm. However, it is important to point out that the methods previously described consider only the membership of nodes into different modules. Since the module structure in this networks contains also topological information, it is possible to approach this comparison from a topological perspective.

To illustrate such comparison, three topology-based comparisons between the Main and Rewired networks are presented. The first one consists on comparing the distribution of the module sizes identified in each network. The second is the comparison of the distribution of embeddedness (Lancichinetti et al. 2010), a measure of the amount of neighbors of each node which belong to its own module. A third one is the comparison of the distribution of modular degree (Ghalmane et al. 2018), which extends on the classical notion of degree centrality to describe both the local and global influence of a node in the network. In Fig. 4 we observe that, based on any of these parameters, the modular structure of each network exhibits differences.

It should be noted that the topology-based comparisons presented here are only a few of the possible options to incorporate this dimension of analysis. In Orman et al. (2012) a more extensive discussion on the subject is found. Nevertheless, the reader should consider using such level of description to more thoroughly evaluate (and convey) the differences in modular structures, and the implications regarding the differences in the underlying biological phenomena.

\section{Comparison of modular structures in networks with non-identical node sets}

In the case of networks where node sets are not identical, it may not be feasible to compare using the aforementioned metrics, as the algorithm implementations are written with identical node sets in mind.

Let us focus on the similarity of modules in each network, in terms of nodes and edges. A matrix of a similarity measure (such as Jaccard index) may be computed, in which each module of the first network is compared to each module of the second network. We provide such matrices for the comparison of the Alternative and Mixed networks against the Main network as Additional file 3. Similarity matrices allow to answer the following questions:

Table 5 Comparison of modular structures detected using the Infomap algorithm Main vs Rewired networks

\begin{tabular}{ll}
\hline Method & Value \\
\hline variation of information & 2.28 \\
normalized mutual information & 0.63 \\
split-join distance & 222.00 \\
Rand index & 0.93 \\
adjusted Rand index & 0.34 \\
\hline
\end{tabular}




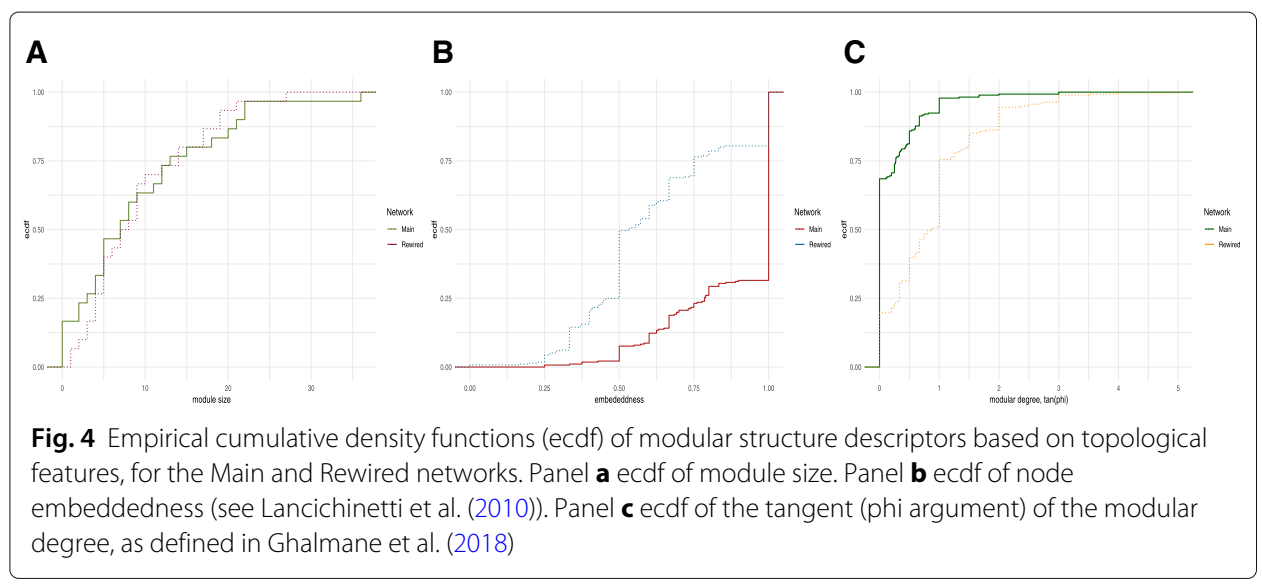

- Whether there are modules in the first network that have some overlap with modules in the second network.

- Whether there are modules in the first network that are perfect matches to modules in the second network.

A global parameter of similarity can be defined to have a descriptor for each network. In this work, we have defined a Similarity Score in terms of the similarity matrix for each pair of networks (see "Methods").

In Table 6 we find the comparison of the Alternative or Mixed networks against the Main network in terms of module node composition. It shows the number of modules in Main that had at least one non-zero overlap with module in the test networks, and the number of modules of Main that perfectly match with a module in the test networks.

In Table 7 we find again a comparison of the Alternative or Mixed networks against the Main network, this time in terms of edge composition. We may notice that in terms of edges, the similarity between the Alternative and Main network is much more penalized.

By comparing the similarities of these partitions, we are able to identify how much are the biological differences of each condition being captured in the modular structures of networks. Furthermore, we gain further information for the discussion of the functional associations identified for each module: for instance, whether functions are being associated to similar or dissimilar modules. The comparison of these modules at the functional level is the topic of the next section.

\section{Functional comparison of modules}

Now the modules will be compared in terms of the associated biological functions identified by an enrichment analysis. This comparison involves answering three complementary questions:

Table 6 Comparison of modular structures detected using the Infomap algorithm between the Main network and the Alternative or Mixed networks, based on module node similarity

\begin{tabular}{llll}
\hline & Similarity_Score & Matching.Modules & Perfect.Matches \\
\hline Alternative & 0.020 & 7 & 0 \\
Mixed & 0.377 & 11 & 9 \\
\hline
\end{tabular}


Table 7 Comparison of modular structures detected using the Infomap algorithm between the Main network and the Alternative or Mixed networks, based on module edge similarity

\begin{tabular}{llll}
\hline & Similarity_Score & Matching.Modules & Perfect.Matches \\
\hline Alternative & 0.001 & 1 & 0 \\
Mixed & 0.367 & 11 & 9 \\
\hline
\end{tabular}

- How similar are the sets of biological functions that are associated to the whole network, through the enrichment of individual modules?

- How similar are the modules found in each network, in terms of the sets of associated biological functions?

- In how many modules is represented each biological process?

When comparing these biological processes, it is important to remember that it is possible for networks composed of different genes to be associated to the same processes and functions. In other words, networks could be functionally comparable even if their node identities are dissimilar. On the other hand, if the genes that integrate the biological functions being evaluated are not present in the networks, these biological functions will not be identified by an enrichment analysis.

\section{Comparison of biological function sets associated to the overall network}

The sets of all biological functions identified in each network are to be compared in terms of similarity. By analyzing the sets of identified biological functions for each network, as shown in Table 8, we can compare and contrast which functions are shared by both networks or uniquely found in each. From the biological perspective, this provides a good starting point to discern which functions or processes may be associated to each condition of interest.

While this comparison is useful as a first approximation, it is soon evident that this is not necessarily considering the modular nature of the network. As such, we will now perform a comparison at the level of individual modules.

\section{Similarity of modules in terms of associated biological functions}

It is possible to compare each module of the network of interest to the modules of another network, in terms of their associated biological functions. A similarity matrix can be calculated for the pair of networks, allowing the identification of modules that are functionally similar in each network, as seen in Table 9.

It is important to consider that, since more than a biological function could be associated to more than one module in a network, it is possible to find, for a given module in a network, more than one functionally similar module in another network.

Table 8 Similarity of enriched processes associated to the overall network

\begin{tabular}{ll}
\hline & Similarity of Enriched Processes, Jaccard Index \\
\hline Main & 1.00 \\
Alternative & 0.50 \\
Mixed & 0.30 \\
Rewired & 0.88 \\
\hline
\end{tabular}


Table 9 Number of modules in networks that have Jaccard index $J=1$ with a module of the Main network

\begin{tabular}{llll}
\hline Module of Main Network & Alternative & Mixed & Rewired \\
\hline 1 & 0 & 3 & 2 \\
2 & 0 & 0 & 0 \\
3 & 0 & 0 & 0 \\
4 & 0 & 0 & 1 \\
5 & 0 & 0 & 3 \\
6 & 0 & 2 & 3 \\
7 & 0 & 2 & 3 \\
8 & 0 & 2 & 1 \\
9 & 0 & 0 & 1 \\
10 & 0 & 0 & 0 \\
11 & 0 & 0 & 2 \\
12 & 0 & 3 & 2 \\
13 & 0 & 3 & 0 \\
14 & 0 & 0 & 1 \\
15 & 0 & 0 & 1 \\
16 & 0 & 0 & 1 \\
17 & 0 & 0 & 0 \\
18 & 0 & 0 & 2 \\
19 & 0 & 3 & 1 \\
20 & 0 & 0 & \\
\hline
\end{tabular}

\section{Number of modules associated to a given biological function}

As it has been mentioned before, it is possible for more than one module to be associated to a given biological function. Therefore, another comparison parameter that can be used to assess the similarities between two biological networks is to evaluate, for each identified biological function, the number of modules that were found to be enriched in that particular function.

It is possible to calculate a distance (for instance, Euclidean) between two networks based on the number of modules associated to each biological function found in the networks, as it is shown in Table 10.

\section{Assessing similarity of modular structures}

The final integration of all comparison parameters in order to assess the similarity of the modular structures in biological networks must be guided by the research question that is to be answered by this comparison. In other words, it would not be possible to establish general thresholds for what is to be accepted as similar or dissimilar.

Table 10 Functional Euclidean Distance of network modules

\begin{tabular}{ll}
\hline & Functional Euclidean Distances \\
\hline Main & 0.00 \\
Alternative & 10.20 \\
Mixed & 8.77 \\
Rewired & 6.00 \\
\hline
\end{tabular}


Furthermore, the objective of the comparison will determine the weight given to a parameter for the definition of comparability. In Table 11, we observe the overall comparison of our three test networks against our Main network. As it has been shown throughout the manuscript, it is possible to determine a quantitative (or semi-quantitative) value of comparison for each network, which can be informative of the points of similarity and divergence between the networks and their associated modular structures.

The alternative network is very dissimilar in terms of node composition. We observe, however, that even then there is some similarity in terms of the overall functions associated to the network. Nevertheless, we find that there are no modules in the Alternative network that precisely reflect the functionality associated to the modules in the Main network. Meanwhile, the mixed network has some overlap in terms of node and edge composition. It exhibits a degree of module topological similarity, and is also functionally closer to the Main network. In relative terms, it is possible to establish that the Mixed network is both topologically and functionally closer to the Main network than the Alternative, which is consistent with the construction of these networks.

The Rewired and Main networks are identical in terms of number of nodes and edges, as well as the identity of nodes, by construction. They were also guaranteed by construction to be different in terms of edges. Since these networks were composed by the same set of nodes, it was possible to compare their modular structures using a well defined parameter of similarity, such as Normalized Mutual Information. Furthermore, we observe that these two networks are very similar in functionality terms.

\section{On the subject of biological comparison versus validation}

An open question remains on how to decide whether a network model is comparable to another network model for the purposes of modular structure comparison. As we have discussed, the two most common situations where a comparison would be necessary are A) the comparison of biological conditions and B) the validation of a biological network model.

Consider that networks that are derived from experimental datasets may or may not be necessarily composed of the same set of nodes, either by technical or biological conditions. In this case, if the experimental settings of each condition are comparable, and the methods for network inference, module detection, and enrichment analysis used in each case are the same, then a comparison in terms of functional similarity is feasible. This setting could also be used for the validation of functional findings, for instance if using a

Table 11 Comparison of the modular structure of the Main network

\begin{tabular}{llll}
\hline Parameter & Alternative & Mixed & Rewired \\
\hline No. Nodes & Lesser & Lesser & Equal \\
No. Edges & Greater & Lesser & Equal \\
Node Similarity $(J)$ & 0.03 & 0.32 & 1.00 \\
Edge Similarity $(J)$ & 0.00 & 0.26 & 0.37 \\
Modularity $(\mathrm{Q})$ & Greater & Greater & Lesser \\
Module Topological Similarity & SimScore edge $=0.01$ & SimScore & edge $=0.30$ \\
Network Functional Similarity $(J)$ & 0.50 & 0.30 & 0.63 \\
Main modules with functionally similar counterparts & $0 / 20$ & $7 / 20$ & $15 / 20$ \\
Functional Euclidian Distance & 10.20 & 8.77 & 6.00 \\
\hline
\end{tabular}


discovery and validation set. An example of such use-case is found in Alcalá-Corona et al. (2018).

Meanwhile, if the objective is the validation of any of the steps of the modular analysis pipeline, it would be important to evaluate the comparability of modular structures and functional enrichment analyses in the context of a network with the same set of nodes and number of edges. Importantly, if a rewiring algorithm is used, the limitations of the implementation should be discussed; for instance, the rewiring algorithm used for the generation of the Rewired network in this work does not preserve the degree distribution, and as such it would not be an adequate model to generate null models for the assessment of functional associations.

\section{Conclusions}

The study of modularity in biological networks provides opportunities to understand the organization of biological phenomena and how these structures shape functionality. In this work, we provide a guideline to quantitatively compare modular structures in biological networks, in topological and functional terms. This provides a basis to identify aspects of network modular structures that may guide the discussion regarding phenotype comparison from a network perspective, as well as some critical points for the validation of network models.

\section{Methods}

All methods used for this work are available at https://github.com/guillermodeandajauregui/ BiologicalModuleComparison

\section{Network Generation}

We generated four networks by acquiring network representations of pathways in KEGG using the Graphite package (Sales et al. 2012). For each of the networks Main, Alternative, and Mixed, the pathways listed in Table 12 were used. In each case, pathways were merged into a single undirected, unweighted network to be analyzed using Igraph (Csardi and Nepusz 2006).

The Rewired network was generated by taking the Main network and using a rewiring algorithm as implemented in Igraph (Csardi and Nepusz 2006), in which the endpoints of edges from network 1 were reconnected randomly, with a uniform rewiring probability of 0.25 .

\section{Module detection}

Modules for the four networks were detected using the Infomap algorithm (Rosvall et al. 2009) as implemented in Igraph. Additionally, modules were detected for the Main

Table 12 Source pathways for the model networks

\begin{tabular}{lll}
\hline Main & Alternative & Mixed \\
\hline Hedgehog signaling pathway & Pentose phosphate pathway & Hedgehog signaling pathway \\
NF-kappa B signaling pathway & Notch signaling pathway & Notch signaling pathway \\
VEGF signaling pathway & mRNA surveillance pathway & Pentose phosphate pathway \\
p53 signaling pathway & TGF-beta signaling pathway & p53 signaling pathway \\
RIG-I-like receptor signaling pathway & IL-17 signaling pathway & \\
\hline
\end{tabular}


network using the Girvan-Newman edge-betweenness algorithm (Girvan and Newman 2002), the Fast-Greedy algorithm (Clauset et al.), the Louvain method (Blondel et al.), the Walktrap method (Latapy and Pons), the spin-glass method (Reichardt and Bornholdt), the leading eigenvector method (Newman), and the label propagation method (Raghavan et al.). A comparison of these algorithms, including a description of each along with suggestions for algorithm selection may be found in Yang et al. (2016); further discussion may be found in Poulin and Théberge (2019).

\section{Module enrichment}

Over-Representation Enrichment analyses for the gene sets of each detected module was performed using hypergeometric testing. This test is equivalent to the one-tailed Fisher's exact test, which assesses the probability of drawing $k$ elements belonging to a set of $K$, by drawing $n$ elements out of a population $N$.

It is widely used as a gene set enrichment tool for gene clustering methods, where the genes in the cluster (or module) form the set of $n$ elements drawn from the $N$ population (usually, the whole set of measured genes). The genes that belong to a given pathway or biological function gene set represent $K$. Finally, the members of the cluster that belong to the pathway or biological function are represented by $k$.

The testing was performed using as implemented in the HTSanalyzer package (Wang et al. 2011). The significance threshold for enrichment was set to be a Benjamini-Hochberg adjusted $p-$ value $<0.05$. For the sake of simplicity in the analysis, it was decided that the list of pathways used for the enrichment analyses would consist of the 10 pathways used to construct networks 1 and 2, plus two additional pathways "Sphingolipid signaling pathway", and "Insulin signaling pathway" that were not originally used for the construction of any network.

\section{Topological network comparison}

The networks were compared in terms of the number of nodes, edges and connected components that composed them. The degree distribution of each network was also obtained. Finally, the similarity of node sets and edge sets of the networks were compared using the Jaccard index.

\section{Jaccard index}

The Jaccard index is a measure of similarity between sets. It considers the sizes of the intersection and union of two sets $A$ and $B$, as follows:

$$
J=\frac{|A \cap B|}{|A \cup B|}
$$

\section{Modular structure comparison}

\section{Evaluation of modular structures}

The modularity score $Q$ (Clauset et al.) was calculated as implemented in the igraph package for R. The topological measures of embeddedness (Lancichinetti et al. 2010) and modular degree (Ghalmane et al. 2018) were implemented following their descriptions in the original references, using the igraph package for $\mathrm{R}$.

\section{Networks with identical node sets}

The modular structures of networks composed of identical node sets were compared using the modularity comparison methods implemented in Igraph. These included the 
Variation of Information (Meilă 2003), Normalized Mutual Information (Danon et al.), split-join distance (van Dongen 2000), and (adjusted) Rand index (Rand 1971; Hubert and Arabie 1985).

\section{Networks with non-identical node sets}

For a pair of networks $G_{1}$ and $G_{2}$, with a modular partition $M_{1}$ and $M_{2}$, we calculate a similarity matrix SimMatrix. For each pair of $M_{i}$ in $G_{1}$ and $M_{j}$ in $G_{2}$, each value $\operatorname{SimMatrix}(i, j)=\operatorname{Similarity}\left(M_{i}, M_{j}\right)$. In this work, the measure of similarity will be the Jaccard index.

We define a Similarity Score in terms of the similarity matrix for each module $M_{i}$ in G1 such that $\operatorname{SimScore}\left(M_{i}\right)=\sum_{j=1}^{q} \frac{J\left(M_{i}, M_{j}\right)}{\left|\left(M_{i} \cap M_{j}\right) \neq \emptyset\right|}$, with $M_{i}=0$ if $\left|\left(M_{i} \cap M_{j}\right) \neq \emptyset\right|=0$.

Furthermore, we then define a a global Similarity Score as $\frac{1}{k} \sum_{i=1}^{k} \operatorname{SimScore}\left(M_{i}\right)$.

\section{Functional comparison}

Network functional similarity

Consider Functions $s_{G}$ to be the set of all biological functions associated to each module of a network G. Functional similarity of two networks is to be calculated as FunctionalSimilarity $=J\left(\right.$ Functions $_{G_{1}}$, Functions $\left._{G_{2}}\right)$

\section{Functional Similarity of Modules}

Consider Functions $s_{i}$ to be the set of biological functions associated to each module $M_{i}$ in a network $G_{1}$, and Functions $s_{j}$ to be the set of biological functions associated to each module $M_{j}$ in a network $G_{2}$. A Functional Similarity Matrix is defined such that FuncSimMatrix $(i, j)=J\left(\right.$ Functions $_{i}$, Functions $\left._{j}\right)$

\section{Number of modules associated to a given biological function}

Consider Functions $G_{G_{1}}$ and Functions $G_{G_{2}}$ to be the set of functions associated through enrichment to $G_{1}$ and $G_{2}$ respectively. Let Functions both $_{\text {bunctions }}=$ Fu $_{G_{1}} \cap$ Functions $_{G_{2}}$. Let $E_{1}$ and $E_{2}$ be $k$-dimensional vectors where each element $k$ of $E_{1}$ and $E_{2}$ is the number of modules in G1 or G2 to which Functions both $_{k}$ is associated. An Euclidian distance between $E_{1}$ and $E_{2}$ can be calculated.

\section{Additional files}

Additional file 1: Figure (PNG) showing the results of a PubMed search for works on networks, clustering, and enrichment, 2000-2019. (PNG 14 kb)

Additional file 2: Table comparing the partitions of the Main network generated by different algorithms, using Adjusted Mutual Information. (PDF $19 \mathrm{~kb}$ )

Additional file 3: Rdata file containing networks, modular structures and enrichment results. (PDF $261 \mathrm{~kb}$ )

Abbreviations

KEGG: Kyoto Encyclopedia of Genes and Genomes; STRING-db: Search Tool for the Retrieval of Interacting Genes database

Acknowledgements

The author would like to thank Enrique Hernández-Lemus and Jesús Espinal-Enríquez, for the discussions leading to this manuscript.

Funding

No funding was allocated for this work. 
Availability of data and materials

ed for the generation and aof networks is available at https:/github.com/guillermodeandajauregui/

BiologicalModuleComparison

Authors' contributions

GDJ developed the work in the present manuscript.

Competing interests

The author declares to have no competing interests.

\section{Publisher's Note}

Springer Nature remains neutral with regard to jurisdictional claims in published maps and institutional affiliations.

\section{Received: 14 November 2018 Accepted: 4 April 2019}

Published online: 18 April 2019

\section{References}

Adamcsek B, Palla G, Farkas IJ, Derényi I, Vicsek T (2006) Cfinder: locating cliques and overlapping modules in biological networks. Bioinformatics (Oxford, England) 22:1021-1023. https://doi.org/10.1093/bioinformatics/btl039

Alcalá-Corona SA, Espinal-Enríquez J, de Anda-Jáuregui G, Hernández-Lemus E (2018) The hierarchical modular structure of her2+ breast cancer network. Front Physiol 9:1423. https://doi.org/10.3389/fphys.2018.01423

Alcalá-Corona SA, de Anda-Jáuregui G, Espinal-Enríquez J, Hernández-Lemus E (2017) Network modularity in breast cancer molecular subtypes. Front Physiol 8:915. https://doi.org/10.3389/fphys.2017.00915

Ames RM, Macpherson JI, Pinney JW, Lovell SC, Robertson DL (2013) Modular biological function is most effectively captured by combining molecular interaction data types. PloS one 8:62670. https://doi.org/10.1371/journal.pone. 0062670

Barabási A-L, Oltvai ZN (2004) Network biology: understanding the cell's functional organization. Nat Rev Gene 5:101-113. https://doi.org/10.1038/nrg1272

Blondel VD, Guillaume J-L, Lambiotte R, Lefebvre E Fast unfolding of communities in large networks. https://doi.org/10. 1088/1742-5468/2008/10/P10008. http://arxiv.org/abs/0803.0476v2. Accessed 30 Mar 2019

Clauset A, Newman MEJ, Moore C Finding community structure in very large networks. https://doi.org/10.1103/ PhysRevE.70.066111. http://arxiv.org/abs/cond-mat/0408187v2. Accessed 30 Mar 2019

Consortium GO (2015) Gene ontology consortium: going forward. Nucleic Acids Res 43:1049-1056. https://doi.org/10. 1093/nar/gku1179

Csardi G, Nepusz T (2006) The igraph software package for complex network research. Inter Journal Complex Systems. 1695

Danon L, Duch J, Diaz-Guilera A, Arenas A Comparing community structure identification. https://doi.org/10.1088/17425468/2005/09/P09008. http://arxiv.org/abs/cond-mat/0505245v2. Accessed 30 Mar 2019

Dobrescu R, Purcarea V (2009) Network based models for biological applications. J Med Life 2:176-184

van Dongen S (2000) A cluster algorithm for graphs. Technical Report INS-R0010. National Research Institute for Mathematics and Computer Science in the Netherlands, Amsterdam

Fortunato S (2010) Community detection in graphs. Phys Rep 486(3):75-174. https://doi.org/10.1016/j.physrep.2009.11. 002

García-Campos MA, Espinal-Enríquez J, Hernández-Lemus E (2015) Pathway analysis: State of the art. Front Phys 6:383. https://doi.org/10.3389/fphys.2015.00383

Ghalmane Z, Hassouni ME, Cherifi C, Cherifi H (2018) Centrality in modular networks. CoRR. http://arxiv.org/abs/1810. 05101

Girvan M, Newman MEJ (2002) Community structure in social and biological networks. PNAS 99(12):7821-7826. https:// doi.org/10.1073/pnas.122653799

Hubert L, Arabie P (1985) Comparing partitions. J Classif 2(1):193-218. https://doi.org/10.1007/BF01908075

Hur J, Schuyler AD, States DJ, Feldman EL (2009) Sciminer: web-based literature mining tool for target identification and functional enrichment analysis. Bioinformatics (Oxford, England) 25:838-840. https://doi.org/10.1093/bioinformatics/ btp049

Jebabli M, Cherifi H, Cherifi C, Hamouda A (2017) Community detection algorithm evaluation with ground-truth data. CoRR. http://arxiv.org/abs/1711.09472

Kanehisa M, Goto S (2000) Kegg: kyoto encyclopedia of genes and genomes. Nucleic Acids Res 28:27-30

Lancichinetti A, Kivelä M, Saramäki J, Fortunato S (2010) Characterizing the community structure of complex networks. PLOS ONE 5(8):1-8. https://doi.org/10.1371/journal.pone.0011976

Langfelder P, Horvath S (2008) Wgcna: an r package for weighted correlation network analysis. BMC bioinformatics 9:559. https://doi.org/10.1186/1471-2105-9-559

Latapy M, Pons P Computing communities in large networks using random walks. http://arxiv.org/abs/cond-mat/ 0412368v1. Accessed 30 Mar 2019

Liu Y, Brossard M, Roqueiro D, Margaritte-Jeannin P, Sarnowski C, Bouzigon E, Demenais F (2017) Sigmod: an exact and efficient method to identify a strongly interconnected disease-associated module in a gene network. Bioinformatics (Oxford, England) 33:1536-1544. https://doi.org/10.1093/bioinformatics/btx004

Lorenz DM, Jeng A, Deem MW (2011) The emergence of modularity in biological systems. Phys Life Rev 8:129-160. https://doi.org/10.1016/j.plrev.2011.02.003

Ma'ayan A (2011) Introduction to network analysis in systems biology. Sci Signal 4:5. https://doi.org/10.1126/scisignal. 2001965

Madhamshettiwar PB, Maetschke SR, Davis MJ, Reverter A, Ragan MA (2012) Gene regulatory network inference: evaluation and application to ovarian cancer allows the prioritization of drug targets. Genome Med 4(5):41. https:// doi.org/10.1186/gm340 
Margolin AA, Nemenman I, Basso K, Wiggins C, Stolovitzky G, Dalla Favera R, Califano A (2006) Aracne: an algorithm for the reconstruction of gene regulatory networks in a mammalian cellular context. BMC bioinformatics 7(Suppl 1):7. https://doi.org/10.1186/1471-2105-7-S1-S7

Meilă M (2003) Comparing clusterings by the variation of information. In: Schölkopf B, Warmuth MK (eds). Learning Theory and Kernel Machines. Springer, Berlin, Heidelberg. pp 173-187

Newman MEJ Finding community structure in networks using the eigenvectors of matrices. https://doi.org/10.1103/ PhysRevE.74.036104. http://arxiv.org/abs/physics/0605087v3. Accessed 30 Mar 2019

Newman MEJ, Girvan M (2004) Finding and evaluating community structure in networks. Phys Rev E 69:026113. https:// doi.org/10.1103/PhysRevE.69.026113

Orman GK, Labatut V, Cherifi H (2012) Comparative evaluation of community detection algorithms: A topological approach. CoRR. http://arxiv.org/abs/1206.4987

Poulin V, Théberge F (2019) Ensemble clustering for graphs. In: Aiello LM, Cherifi C, Cherifi H, Lambiotte R, Lió P, Rocha LM (eds). Complex Networks and Their Applications VIl. Springer, Cham. pp 231-243

Raghavan UN, Albert R, Kumara S Near linear time algorithm to detect community structures in large-scale networks. https://doi.org/10.1103/PhysRevE.76.036106. http://arxiv.org/abs/0709.2938v1. Accessed 30 Mar 2019

Rand WM (1971) Objective criteria for the evaluation of clustering methods. J Am Stat Assoc 66(336):846-850. https://doi. org/10.1080/01621459.1971.10482356. http://arxiv.org/abs/https://www.tandfonline.com/doi/pdf/10.1080/ 01621459.1971.10482356. https://www.tandfonline.com/doi/pdf/10.1080/01621459.1971.10482356

Reichardt J, Bornholdt S Statistical mechanics of community detection. https://doi.org/10.1103/PhysRevE.74.016110. http://arxiv.org/abs/cond-mat/0603718v1. Accessed 30 Mar 2019

Rosvall M, Axelsson D, Bergstrom CT (2009) The map equation. Eur Phys J Spec Top 178(1):13-23

Sales G, Calura E, Cavalieri D, Romualdi C (2012) graphite - a bioconductor package to convert pathway topology to gene network. BMC bioinformatics 13:20. https://doi.org/10.1186/1471-2105-13-20

Szklarczyk D, Morris JH, Cook H, Kuhn M, Wyder S, Simonovic M, Santos A, Doncheva NT, Roth A, Bork P, Jensen L, von Mering C (2017) The string database in 2017: quality-controlled protein-protein association networks, made broadly accessible. Nucleic Acids Res 45:362-368. https://doi.org/10.1093/nar/gkw937

Vinh NX, Epps J, Bailey J (2009) Information theoretic measures for clusterings comparison: Is a correction for chance necessary? In: Proceedings of the 26th Annual International Conference on Machine Learning. ICML '09. ACM, New York. pp 1073-1080. https://doi.org/10.1145/1553374.1553511

Vinh NX, Epps J, Bailey J (2010) Information theoretic measures for clusterings comparison: Variants, properties, normalization and correction for chance. J Mach Learn Res 11:2837-2854

Wang X, Terfve C, Rose JC, Markowetz F (2011) Htsanalyzer: an r/bioconductor package for integrated network analysis of high-throughput screens. Bioinformatics 27(6):879-880

Yang Z, Algesheimer R, Tessone CJ (2016) A comparative analysis of community detection algorithms on artificial networks. Scientific Reports 6

\section{Submit your manuscript to a SpringerOpen ${ }^{\circ}$ journal and benefit from:}

- Convenient online submission

- Rigorous peer review

- Open access: articles freely available online

- High visibility within the field

- Retaining the copyright to your article

Submit your next manuscript at $\boldsymbol{\triangleright}$ springeropen.com 